\title{
Magnetic oscillations in dense cold quark matter with four-fermion interactions
}

\author{
D. Ebert* \\ Theory Division, CERN, CH 1211 Geneva 23, Switzerland \\ and Institut für Physik, Humboldt-Universität, D-10115 Berlin, Germany \\ K. G. Klimenko ${ }^{\dagger}$ \\ Institute for High Energy Physics, 142284 Protvino, Moscow Region, Russia \\ M. A. Vdovichenko and A. S. Vshivtsev \\ Moscow Institute of Radio-Engineering, Electronics and Automatic Systems, 117454 Moscow, Russia
}

(Received 11 May 1999; published 16 December 1999)

\begin{abstract}
The phase structures of Nambu-Jona-Lasinio models with one or two flavors are investigated at nonzero values of $\mu$ and $H$, where $H$ is an external magnetic field and $\mu$ is the chemical potential. In the phase portraits of both models there arise infinitely many massless chirally symmetric phases, as well as massive ones with spontaneously broken chiral invariance, reflecting the existence of infinitely many Landau levels. Phase transitions of first and second orders and a lot of tricritical points have been shown to exist in phase diagrams. In the massless case, such a phase structure leads unavoidably to the standard van Alphen-de Haas magnetic oscillations of some thermodynamical quantities, including magnetization, pressure and particle density. In the massive case we find an oscillating behavior not only for thermodynamical quantities, but also for a dynamical quantity as the quark mass. In addition, in this case we have nonstandard, i.e., nonperiodic, magnetic oscillations, since the frequency of oscillations is an $H$-dependent quantity.
\end{abstract}

PACS number(s): 11.30.Qc, 12.38.Mh, 21.65.+f, 71.27.+a

\section{INTRODUCTION}

The exploration of strongly interacting matter at high density and in the presence of external electromagnetic fields is of fundamental interest and has potential applications to the quark-gluon plasma and heavy-ion collisions, to cosmology and astrophysics of neutron stars. Recently, some aspects of this problem were considered in [1], where it was pointed out that in QCD at high density a new phase with color superconductivity might exist. The influence of external magnetic fields on the QCD vacuum was, for example, studied in [2].

Our goal is to investigate the properties of the strongly interacting cold quark matter in the presence of both the external magnetic field $H$ and the nonzero chemical potential $\mu$. The subject is closely related to magnetic oscillations of different physical quantities. In this connection we should remember that the van Alphen-de Haas effect (oscillations of the magnetization) was predicted for the first time by Landau and then experimentally observed in some nonrelativistic systems (in metals) more than 60 years ago $[3,4]$. At present, a lot of the attention of researchers dealing with magnetic oscillations is focused on relativistic condensed matter systems (mainly on QED at $\mu, H \neq 0$ ), since the results of these studies may be applied to cosmology, astrophysics, and highenergy physics $[5,6]$.

It is well known that up to now the consideration of QCD at $\mu, H \neq 0$ is a difficult problem. This is partly due to the

\footnotetext{
*Email address: dietmar.ebert@cern.ch

${ }^{\dagger}$ Email address: kklim@mx.ihep.su

${ }^{*}$ Deceased.
}

fact that numerical lattice simulations at $\mu \neq 0$ have not been able to overcome problems associated with the complex part of the fermionic determinant. Moreover, the incorporation of a magnetic field into lattice gauge calculations is not elaborated sufficiently, either. For these reasons, when considering quark matter at $\mu, H \neq 0$, many authors prefer to deal with adequate models (e.g., with the MIT bag model [7]), rather than with QCD.

In the present paper we shall study the above problem in the framework of some specific QCD-like quark models. Namely, we shall investigate the influence of an external magnetic field and chemical potential on the vacuum structure of Nambu-Jona-Lasinio (NJL) models containing fourfermion interactions $[8,9]$. The simplest one, denoted as model I, refers to the one-flavor case and is presented by the Lagrangian

$$
L_{1}=\bar{q}_{k} i \hat{\partial} q_{k}+\frac{G}{2 N_{c}}\left[\left(\bar{q}_{k} q_{k}\right)^{2}+\left(\bar{q}_{k} i \gamma_{5} q_{k}\right)^{2}\right]
$$

where all quark fields belong to the fundamental multiplet of the color $\mathrm{SU}\left(N_{c}\right)$ group (here the summation over the color index $k=1, \ldots, N_{c}$ is implied). Obviously, $L_{1}$ is invariant under (global) $\mathrm{SU}\left(N_{c}\right)$ and $\mathrm{U}(1)_{V}$ transformations as well as continuous $\mathrm{U}(1)_{A}$ chiral transformations

$$
q_{k} \rightarrow e^{i \theta \gamma_{5}} q_{k} \quad\left(k=1, \ldots, N_{c}\right) .
$$

The second, more realistic case considered here and referred to as model II is a two-flavor NJL model whose Lagrangian has the form 


$$
L_{2}=\bar{q} i \hat{\partial} q+\frac{G}{2 N_{c}}\left[(\bar{q} q)^{2}+\left(\bar{q} i \gamma^{5} \vec{\tau} q\right)^{2}\right]
$$

where $q$ is a flavor isodoublet and color- $N_{c}$-plet quark field and $\vec{\tau}$ are isospin Pauli matrices [in Eq. (3) and below, flavor and color indices of the quark field $q$ are now suppressed]. The Lagrangian $L_{2}$ is invariant under (global) $\mathrm{U}(2)_{f}$ $\times \mathrm{SU}\left(N_{c}\right)$ as well as under chiral $\mathrm{U}(2)_{L} \times \mathrm{U}(2)_{R}$ groups.

NJL models were proposed as a good laboratory for investigating the nonperturbative phenomenon of dynamical chiral symmetry breaking (DCSB), which occurs in the physics of strong interactions, as well as for describing the lowenergy sector of QCD (see, e.g., papers [10-12] and references therein). Since there are no closed physical systems in nature, the influence of different external factors on the DCSB mechanism is of great interest. In this relation, special attention has been paid to the analysis of the vacuum structure of NJL-type models at nonzero temperature and chemical potential $[13,14]$, in the presence of external (chromo-)magnetic fields [15-17], with allowance for curvature and nontrivial space-time topology $[18,19]$. The combined influence of external electromagnetic and gravitational fields on the DCSB effect in four-fermion field theories was investigated in [20,21].

In the present paper the phase structures and related oscillating effects of the above-mentioned NJL models are considered at $\mu, H \neq 0$ in the leading order of the large $N_{c}$-approximation. We will show that, here, the set of oscillating physical parameters in NJL models is richer than in QED at $\mu, H \neq 0$. Besides, in the NJL models, in contrast to QED and similar to some condensed-matter materials, there exist nonperiodic magnetic oscillations.

\section{NJL MODELS AT $\mu \neq 0$ AND $H=0$}

First of all let us prepare the basis for the investigations in the following sections and consider in detail the phase structure of the model I at nonzero chemical potential $\mu \neq 0$ and $H=0$.

Recall some well-known vacuum properties of the theory (1) at $\mu=0$. The introduction of an intermediate quarkmeson Lagrangian

$$
\tilde{L}_{1}=\bar{q} i \hat{\partial} q-\bar{q}\left(\sigma_{1}+i \sigma_{2} \gamma_{5}\right) q-\frac{N_{c}}{2 G}\left(\sigma_{1}^{2}+\sigma_{2}^{2}\right)
$$

greatly facilitates the problem under consideration. [In Eq. (4) and other formulas below we have omitted the fermionic index $k$ for simplicity.] Clearly, using the equations of motion for the bosonic fields $\sigma_{1,2}$, the theory in Eq. (4) is equivalent to that in Eq. (1). From Eq. (4) we obtain the one-loop expression for the effective action

$$
\exp \left[i N_{c} S_{e f f}\left(\sigma_{1,2}\right)\right]=\int D \bar{q} D q \exp \left(i \int \tilde{L}_{1} d^{4} x\right)
$$

where

$$
S_{e f f}\left(\sigma_{1,2}\right)=-\int d^{4} x \frac{\sigma_{1}^{2}+\sigma_{2}^{2}}{2 G}-i \ln \operatorname{det}\left(i \hat{\partial}-\sigma_{1}-i \gamma_{5} \sigma_{2}\right)
$$

Assuming that in this formula $\sigma_{1,2}$ are independent of spacetime points, we have by definition

$$
\begin{gathered}
S_{e f f}\left(\sigma_{1,2}\right)=-V_{0}\left(\sigma_{1,2}\right) \int d^{4} x, \\
V_{0}\left(\sigma_{1,2}\right)=\frac{\Sigma^{2}}{2 G}+2 i \int \frac{d^{4} p}{(2 \pi)^{4}} \ln \left(\Sigma^{2}-p^{2}\right) \equiv V_{0}(\Sigma),
\end{gathered}
$$

where $\Sigma=\sqrt{\sigma_{1}^{2}+\sigma_{2}^{2}}$. Next, by introducing in Eq. (5) the Euclidean metric $\left(p_{0} \rightarrow i p_{0}\right)$ and cutting off the range of integration $\left(p^{2} \leqslant \Lambda^{2}\right)$, we obtain

$$
\begin{aligned}
V_{0}(\Sigma)= & \frac{\Sigma^{2}}{2 G}-\frac{1}{16 \pi^{2}}\left[\Lambda^{4} \ln \left(1+\frac{\Sigma^{2}}{\Lambda^{2}}\right)\right. \\
& \left.+\Lambda^{2} \Sigma^{2}-\Sigma^{4} \ln \left(1+\frac{\Lambda^{2}}{\Sigma^{2}}\right)\right] .
\end{aligned}
$$

The stationarity equation for the effective potential (6) has the form

$$
\begin{aligned}
\frac{\partial V_{0}(\Sigma)}{\partial \Sigma}= & =\frac{\Sigma}{4 \pi^{2}} \\
& \times\left[\frac{4 \pi^{2}}{G}-\Lambda^{2}+\Sigma^{2} \ln \left(1+\frac{\Lambda^{2}}{\Sigma^{2}}\right)\right] \equiv \frac{\Sigma}{4 \pi^{2}} F(\Sigma) .
\end{aligned}
$$

Now one can easily see that at $G<G_{c}=4 \pi^{2} / \Lambda^{2}$, Eq. (7) has no solutions apart from $\Sigma=0$. Hence, in this case fermions are massless, and chiral invariance (2) is not broken.

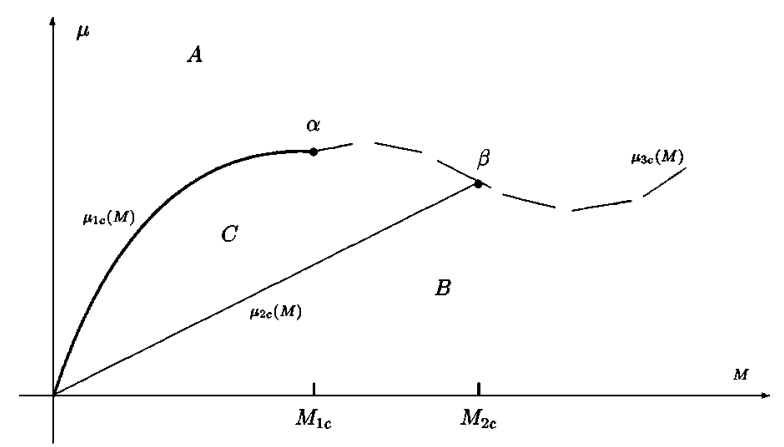

FIG. 1. Phase portrait of the NJL model at nonzero $\mu$ and for arbitrary values of the fermion mass $M$. Phases $B$ and $C$ are massive and nonsymmetric, $A$ is a chirally symmetric phase. Here $\mu_{2 c}(M)$ $=M, \quad \mu_{1 c}(M)=\sqrt{\frac{1}{2} M^{2} \ln \left(1+\Lambda^{2} / M^{2}\right)}, \quad \mu_{3 c}(M)$ is the solution of the equation $V_{\mu}(0)=V_{\mu}(M), M_{2 c}=\Lambda /(2.21 \ldots), M_{1 c}$ is the solution of the equation $\mu_{1 c}^{2}\left(M_{1 c}\right)=\Lambda^{2} /(4 e)$. In phase $B$ the particle density in the ground state is equal to zero, whereas in phase $C$ it is nonzero. Solid and dashed lines represent critical curves of secondand first-order phase transitions, respectively; $\alpha$ and $\beta$ denote tricritical points. 


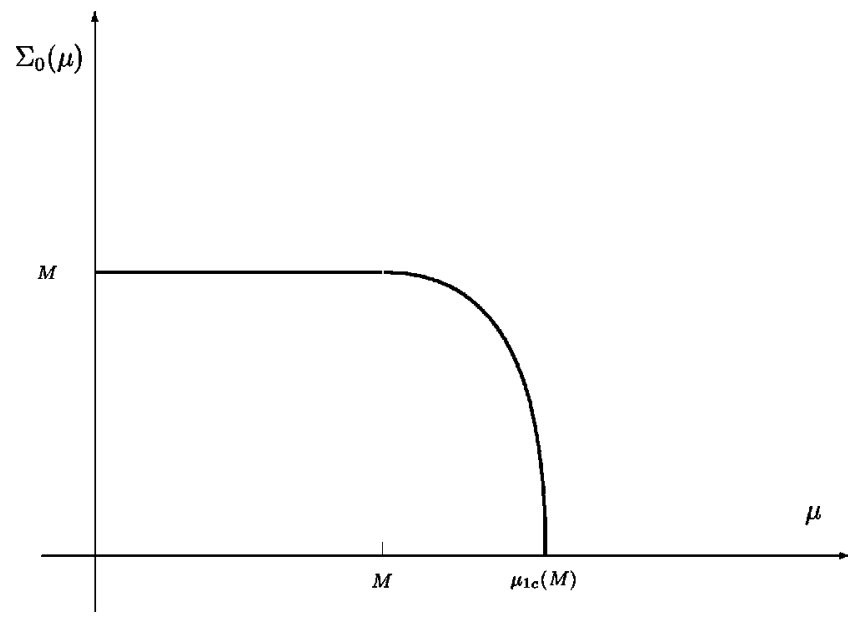

FIG. 2. The behavior of the dynamical quark mass $\Sigma_{0}(\mu)$ as a function of $\mu$ for the case $M<M_{1 c}$ and $H=0$ within the framework of model I.

If $G>G_{c}$, then Eq. (7) has one nontrivial solution, $\Sigma_{0}(G, \Lambda) \neq 0$, such that $F\left(\Sigma_{0}\right)=0$. In this case $\Sigma_{0}$ is a point of global minimum for the potential $V_{0}(\Sigma)$. This means that spontaneous breaking of the symmetry (2) takes place, since $\Sigma_{0}$ is proportional to the chiral symmetry breaking order parameter $\langle q \bar{q}\rangle$, which at $\Sigma_{0} \neq 0$ is not equal to zero. Moreover, fermions acquire a mass $M \equiv \Sigma_{0}(G, \Lambda)$.

Let us now consider the case where $\mu>0$ and the temperature $T \neq 0$. In this case, the effective potential $V_{\mu T}(\Sigma)$ can be found if the measure of integration in Eq. (5) is transformed in the standard way according to the rule [22]

$$
\int \frac{d p_{0}}{2 \pi} \rightarrow i T \sum_{n=-\infty}^{\infty}, \quad p_{0} \rightarrow i \pi T(2 n+1)+\mu .
$$

Summing there over $n$ and letting the temperature in the obtained expression tend to zero, we obtain

$$
\begin{aligned}
V_{\mu}(\Sigma)= & V_{0}(\Sigma)-2 \int \frac{d^{3} p}{(2 \pi)^{3}} \\
& \times \theta\left(\mu-\sqrt{\Sigma^{2}+p^{2}}\right)\left(\mu-\sqrt{\Sigma^{2}+p^{2}}\right),
\end{aligned}
$$

where $\theta(x)$ is the step function. Finally, by performing the momentum integration, we find

$$
\begin{aligned}
V_{\mu}(\Sigma)= & V_{0}(\Sigma)-\frac{\theta(\mu-\Sigma)}{16 \pi^{2}}\left\{\frac{10}{3} \mu\left(\mu^{2}-\Sigma^{2}\right)^{3 / 2}\right. \\
& \left.-2 \mu^{3} \sqrt{\mu^{2}-\Sigma^{2}}+\Sigma^{4} \ln \left[\left(\mu+\sqrt{\mu^{2}-\Sigma^{2}}\right)^{2} / \Sigma^{2}\right]\right\} .
\end{aligned}
$$

It follows from Eq. (8) that, in the case $G<G_{c}$ and at arbitrary values of the chemical potential, the chiral symmetry (2) is not broken. However, at $G>G_{c}$ the model has a rich phase structure, which is presented in Fig. 1 in terms of $\mu$ and $M$. [At $G>G_{c}$ one can use the fermionic mass $M$ as an independent parameter of the theory. The three quantities $G$,
$M$, and $\Lambda$ are connected by Eq. (7).] In this figure the solid and dashed lines represent the critical curves of the secondand first-order phase transitions, respectively. Furthermore, there are two tricritical points, ${ }^{1} \alpha$ and $\beta$, two massive phases $B$ and $C$ with spontaneously broken chiral invariance as well as the symmetric massless phase $A$ in the phase portrait of the NJL model I (detailed calculations of the vacuum structure of this NJL model can be found in [14]). In the present model the dynamical quark mass $\Sigma_{0}(\mu)$, given by the global minimum of the potential $V_{\mu}(\Sigma)$ as a function of $\mu$, behaves as depicted in Fig. 2.

We should note that the phase transition from $B$ to $C$ is the quark-matter analogue of the so-called insulator-metal phase transition in condensed-matter physics. This is due to the fact that, in the vacuum of phase $B$, the particle density (the analogue of conductivity electron density in condensed materials) is zero, while in the vacuum of phase $C$ there arises a nonzero density of charged particles, so that it looks like a Fermi-liquid ground state of metals.

To investigate the vacuum properties of the two-flavor NJL model (model II) it is again convenient to employ, instead of the quark Lagrangian (3), the equivalent quarkmeson Lagrangian

$$
\tilde{L}_{2}=\bar{q} i \gamma^{\mu} \partial_{\mu} q-\bar{q}\left(\sigma+i \gamma^{5} \vec{\tau} \vec{\pi}\right) q-\frac{N_{c}}{2 G}\left(\sigma^{2}+\vec{\pi}^{2}\right)
$$

Using calculations similar to the case with model I, one can find the effective potentials for $\mu=0$ and $\mu \neq 0$ expressed in terms of meson fields $\sigma, \vec{\pi}$. These potentials have the form of the potentials (6) and (8) for model I, respectively, with the exception that the factor $\left(16 \pi^{2}\right)^{-1}$ in Eqs. (6) and (8) has to be replaced by $\left(8 \pi^{2}\right)^{-1}$. Moreover, ${ }^{2}$ in the case under consideration $\Sigma^{2}=\sigma^{2}+\vec{\pi}^{2}$. It follows from this similarity that the phase structure and the phase portrait of model II are qualitatively the same as those of model I (see Fig. 1).

Note that our investigation here is based on "standard" NJL models containing only color-singlet $(q \bar{q})$ interactions. Their path-integral bosonization leads to a single order parameter given by the quark condensate. In the recent literature, one has further considered QCD-motivated extended NJL models as arising from the Fierz transformation of current-current interactions mediated by (nonperturbative) gluon exchange. Such enlarged NJL models, which include, besides usual $(q \bar{q})$ interactions, additional $(q q)$-interaction channels, contain then two order parameters: the usual quark

\footnotetext{
${ }^{1} \mathrm{~A}$ point of the phase diagram is called a tricritical one if, in an arbitrarily small vicinity of it, there are first- as well as second-order phase transitions.

${ }^{2}$ Chiral symmetry of the two-flavor NJL model (3) is realized in the space of meson fields $(\sigma, \vec{\pi})$ as the rotation group $O(4)$, which leaves the "length" of the particle vector $(\sigma, \vec{\pi})$ invariant. Hence, all effective potentials of this model depend on the single variable $\Sigma=\sqrt{\sigma^{2}+\vec{\pi}^{2}}$. In the following we choose $\vec{\pi}=0$, assuming the absence of a pion condensate.
} 
condensate $\langle q \bar{q}\rangle$ and, in addition, the diquark condensate $\langle q q\rangle$ of quark Cooper pairs [for early work on extended NJL-type of models including additional $(q q)$-interaction channels see, e.g., Ref. [23]]. For such models one has, in particular, shown that at sufficiently large values of the chemical potential there might arise a new phase of color superconductivity signaled by a nonvanishing diquark condensate $\langle q q\rangle$. This fact was, e.g., established in [24] for an extended version of the one-flavor model I and in [25] for an extended model II. Since in the present paper we are interested in the consideration of the chiral symmetry breaking alone, we have restricted our study to the above form of standard NJL models, leaving aside the possibility of additional $(q q)$ interactions and of Cooper pairing of quarks.

\section{PHASE STRUCTURE OF MODEL I AT $\mu \neq 0$ AND $H \neq 0$}

In the present section we shall study vacuum magnetic properties of NJL systems. For model I at $\mu=0$, this problem was considered in $[15,17]$. It was shown in [15] that at $G>G_{c}$ the chiral symmetry is spontaneously broken for arbitrary values of the external magnetic field $H$, including the case $H=0$. At $G<G_{c}$ the NJL model I has a symmetric vacuum at $H=0$. However, if an external (arbitrarily small) magnetic field is switched on, then for all $G \in\left(0, G_{c}\right)$ there is a spontaneous breaking of the initial $\mathrm{U}(1)_{A}$ symmetry (2) [17]. This is the so-called effect of dynamical chiral symmetry breaking (DCSB) catalysis by an external magnetic field. [This effect was observed for the first time in the framework of a $(2+1)$-dimensional Gross-Neveu model in [26] and was then explained in [27]. Now this effect is under intensive investigations since it has a wide range of possible applications in physics. $\left.{ }^{3}\right]$

Let us recall some aspects of the problem at $\mu=0, H \neq 0$. In order to find in this case the effective potential $V_{H}(\Sigma)$ of the NJL model I, gauged by an external magnetic field according to $\partial_{\mu} \rightarrow D_{\mu}=\partial_{\mu}-i e A_{\mu}, A_{\mu}=\delta_{\mu 2} x_{1} H$, one can use the well-known proper-time method [29] or momentum space calculations [30], which in the leading order of the $1 / N_{c}$ expansion gives the following expression:

$$
V_{H}(\Sigma)=\frac{H^{2}}{2}+\frac{\Sigma^{2}}{2 G}+\frac{e H}{8 \pi^{2}} \int_{0}^{\infty} \frac{d s}{s^{2}} \exp \left(-s \Sigma^{2}\right) \operatorname{coth}(e H s) .
$$

In this formula $e$ has a positive value. It is useful to rearrange this expression in the form

$$
V_{H}(\Sigma)=V_{0}(\Sigma)+Z(\Sigma)+\widetilde{V}_{H}(\Sigma)
$$

where

$$
V_{0}(\Sigma)=\frac{\Sigma^{2}}{2 G}+\frac{1}{8 \pi^{2}} \int_{0}^{\infty} \frac{d s}{s^{3}} \exp \left(-s \Sigma^{2}\right)
$$

\footnotetext{
${ }^{3}$ Some recent references on this subject are presented in [28].
}

$$
\begin{aligned}
Z(\Sigma)= & \frac{H^{2}}{2}+\frac{(e H)^{2}}{24 \pi^{2}} \int_{0}^{\infty} \frac{d s}{s} \exp \left(-s \Sigma^{2}\right), \\
\widetilde{V}_{H}(\Sigma)= & \frac{1}{8 \pi^{2}} \int_{0}^{\infty} \frac{d s}{s^{3}} \exp \left(-s \Sigma^{2}\right) \\
& \times\left((e H s) \operatorname{coth}(e H s)-1-\frac{(e H s)^{2}}{3}\right),
\end{aligned}
$$

thereby isolating the contributions of the matter, the field, and the electromagnetic interaction energy densities explicitly. The potential $V_{0}(\Sigma)$ in Eq. (11) is up to an unimportant (infinite) additive constant not depending on $\Sigma$, equal to expression (5). Hence, the ultraviolet (UV) regularized expression for it looks like Eq. (6).

The integral of the function $Z(\Sigma)$ is also UV divergent, so we need to regularize it. The simplest possibility is to cut it off at the lower boundary, which yields

$$
Z(\Sigma)=\frac{H^{2}}{2}-\frac{(e H)^{2}}{24 \pi^{2}}\left(\ln \frac{\Sigma^{2}}{\Lambda^{2}}+\gamma\right),
$$

$\gamma$ being the Euler constant. Clearly, the last term in Eq. (12) contributes to the renormalization of the magnetic field and electric charge, in a way similar to what occurs in quantum electrodynamics [29].

The potential $\widetilde{V}_{H}(\Sigma)$ in Eq. (11) has no UV divergences, so it is easily calculated with the help of integral tables [31]. The final expression for $V_{H}(\Sigma)$ in terms of renormalized quantities is then given by

$$
\begin{aligned}
V_{H}(\Sigma)= & \frac{H^{2}}{2}+V_{0}(\Sigma)-\frac{(e H)^{2}}{2 \pi^{2}} \\
& \times\left(\zeta^{\prime}(-1, x)-\frac{1}{2}\left[x^{2}-x\right] \ln x+\frac{x^{2}}{4}\right),
\end{aligned}
$$

where $x=\Sigma^{2} /(2 e H), \zeta(\nu, x)$ is the generalized Riemann zeta function and $\zeta^{\prime}(-1, x)=d \zeta(\nu, x) /\left.d \nu\right|_{\nu=-1}$. The global minimum point of this function is the solution of the stationarity equation

$$
\frac{\partial}{\partial \Sigma} V_{H}(\Sigma)=\frac{\Sigma}{4 \pi^{2}}\{F(\Sigma)-I(\Sigma)\}=0
$$

where $F(\Sigma)$ is given in $(7)$, and

$$
I(\Sigma)=2 e H\left\{\ln \Gamma(x)-\frac{1}{2} \ln (2 \pi)+x-\frac{1}{2}(2 x-1) \ln x\right\}
$$

$$
=\int_{0}^{\infty} \frac{d s}{s^{2}} \exp \left(-s \Sigma^{2}\right)[e H s \operatorname{coth}(e H s)-1] .
$$

One can easily see that there exists, for arbitrary fixed values of $H, G$, only one nontrivial solution $\Sigma_{0}(H)$ of Eq. (14), which is the global minimum point of $V_{H}(\Sigma)$. There, $\Sigma_{0}(H)$ is a monotonically increasing function of $H$, and at $H \rightarrow \infty$ 


$$
\Sigma_{0}(H) \approx \frac{e H}{\pi} \sqrt{\frac{G}{12}}
$$

However, at $H \rightarrow 0$

$$
\Sigma_{0}^{2}(H) \approx \begin{cases}\frac{e H}{\pi} \exp \left\{-\frac{1}{e H}\left(\frac{4 \pi^{2}}{G}-\Lambda^{2}\right)\right\} & \text { if } G<G_{c}, \\ M^{2} & \text { if } G>G_{c} .\end{cases}
$$

So, at $G<G_{c}$ and $H=0$ the NJL vacuum is chirally symmetric, but an arbitrarily small value of the external magnetic field $H$ induces DCSB, and fermions acquire a nonzero mass $\Sigma_{0}(H)$ (the magnetic catalysis effect of DCSB).

Now let us consider the more general case, when $H \neq 0$ and $\mu \neq 0$. In one of our previous papers [32] an effective potential of a 3D Gross-Neveu model at nonzero $H, \mu$, and $T$ was obtained. Similarly, one can find the effective potential in the NJL model I at $H, T, \mu \neq 0$ :

$$
\begin{aligned}
V_{H \mu T}(\Sigma)= & V_{H}(\Sigma)-\frac{T e H}{4 \pi^{2}} \sum_{k=0}^{\infty} \alpha_{k} \\
& \times \int_{-\infty}^{\infty} d p \ln \left\{\left[1+e^{-\beta\left(\varepsilon_{k}+\mu\right)}\right]\left[1+e^{-\beta\left(\varepsilon_{k}-\mu\right)}\right]\right\},
\end{aligned}
$$

where $\beta=1 / T, \alpha_{k}=2-\delta_{0 k}, \varepsilon_{k}=\sqrt{\Sigma^{2}+p^{2}+2 e H k}$, with $k=0,1,2, \ldots$ denoting Landau levels, and the function $V_{H}(\Sigma)$ is given in Eq. (13). If we let the temperature in Eq. (18) tend to zero, we obtain the effective potential of the NJL model I at $H, \mu \neq 0$ :

$$
V_{H \mu}(\Sigma)=V_{H}(\Sigma)-\frac{e H}{4 \pi^{2}} \sum_{k=0}^{\infty} \alpha_{k} \int_{-\infty}^{\infty} d p\left(\mu-\varepsilon_{k}\right) \theta\left(\mu-\varepsilon_{k}\right)
$$

which, by performing the integration, can easily be cast into the form

$$
\begin{aligned}
V_{H \mu}(\Sigma)= & V_{H}(\Sigma)-\frac{e H}{4 \pi^{2}} \sum_{k=0}^{\infty} \alpha_{k} \theta\left(\mu-s_{k}\right) \\
& \times\left(\mu \sqrt{\mu^{2}-s_{k}^{2}}-s_{k}^{2} \ln \left[\frac{\mu+\sqrt{\mu^{2}-s_{k}^{2}}}{s_{k}}\right]\right),
\end{aligned}
$$

where $s_{k}=\sqrt{\Sigma^{2}+2 e H k}$. Finally, let us present the stationarity equation for the potential (20):

$$
\frac{\partial}{\partial \Sigma} V_{H \mu}(\Sigma) \equiv \frac{\Sigma}{4 \pi^{2}} \phi(\Sigma)=\frac{\Sigma}{4 \pi^{2}}\left(F(\Sigma)-I(\Sigma)+2 e H \sum_{k=0}^{\infty} \alpha_{k} \theta\left(\mu-s_{k}\right) \ln \left[\frac{\mu+\sqrt{\mu^{2}-s_{k}^{2}}}{s_{k}}\right]\right)=0 .
$$

In order to get a phase portrait of the model under consideration we should find a one-to-one correspondence between points of the $(\mu, H)$ plane and the global minimum points of the function (20), i.e., by solving Eq. (21) we should find the global minimum $\Sigma(\mu, H)$ of the potential (20) and then study its properties as a function of $(\mu, H)$.

\section{A. The case $G<G_{c}$. Magnetic catalysis and chemical potential}

In order to greatly simplify this problem, let us divide the $(\mu, H)$ plane into a set of regions $\omega_{k}$ :

$$
\begin{gathered}
(\mu, H)=\bigcup_{k=0}^{\infty} \omega_{k}, \\
\omega_{k}=\left\{(\mu, H): 2 e H k \leqslant \mu^{2} \leqslant 2 e H(k+1)\right\} .
\end{gathered}
$$

In the $\omega_{0}$ region only the first term from the series in Eqs. (20) and (21) is nonvanishing. So, one can find that, for the points $(\mu, H) \in \omega_{0}$, which are above the line $l=\{(\mu, H): \mu$ $\left.=\Sigma_{0}(H)\right\}$, the global minimum is at the point $\Sigma=0$. Just under the curve $l$ the point $\Sigma=\Sigma_{0}(H)$ is a local minimum of the potential (20), whereas $\Sigma=\Sigma_{0}(H)$ becomes a global minimum, when $(\mu, H)$ lies under the curve $\mu=\mu_{c}(H)$, which is defined by the following equation:

$$
V_{H \mu}(0)=V_{H \mu}\left(\Sigma_{0}(H)\right) \text {. }
$$

Evidently, the line $\mu=\mu_{c}(H)$ is the critical curve of firstorder phase transitions. In the $\omega_{0}$ region Eq. (23) is easily solved as

$$
\mu_{c}(H)=\frac{2 \pi}{\sqrt{e H}}\left[V_{H}(0)-V_{H}\left(\Sigma_{0}(H)\right)\right]^{1 / 2} .
$$

Using the asymptotics (17) of the solution $\Sigma_{0}(H)$ at $H \rightarrow 0$, we find the following behavior of $\mu_{c}(H)$ at $H \rightarrow 0$ :

$$
\mu_{c}(H) \approx \sqrt{\frac{e H}{2 \pi}} \exp \left\{-\frac{1}{2 e H}\left(\frac{4 \pi^{2}}{G}-\Lambda^{2}\right)\right\} .
$$

Hence, we have shown that at $\mu>\mu_{c}(H) \quad\left(G<G_{c}\right)$ there exists a massless symmetric phase of the NJL model [numerical investigations of Eqs. (20) and (21) give us a zero global minimum point for the potential $V_{H \mu}(\Sigma)$ in other regions $\omega_{1}, \omega_{2}, \ldots$ as well]. The external magnetic field 
ceases to induce the DCSB at $\mu>\mu_{c}(H)$ [or at sufficiently small values of the magnetic field $H<H_{c}(\mu)$, where $H_{c}(\mu)$ is the inverse function of $\left.\mu_{c}(H)\right]$. However, under the critical curve (24) [or at $H>H_{c}(\mu)$ ], owing to the presence of an external magnetic field, the chiral symmetry is spontaneously broken. Here the magnetic field induces a dynamical fermion mass $\Sigma_{0}(H)$, which has a $\mu$-independent value.

Lastly, we should also remark that in the NJL model (1) the magnetic catalysis effect takes place only in the phase with zero particle density, i.e., at $\mu<\mu_{c}(H)$. If $\mu>\mu_{c}(H)$, we have the symmetric phase with nonzero particle density, but here the magnetic field cannot induce DCSB.

\section{B. The case $G<G_{c}$. Infinite cascade of massless phases}

In the previous subsection we have shown that the points ( $\mu, H$ ), lying above the critical curve $\mu=\mu_{c}(H)$, correspond to the chirally symmetric ground state of the NJL model. Fermionic excitations of this vacuum have zero masses. At first sight, it might seem that the properties of this symmetric vacuum are slightly varied, when parameters $\mu$ and $H$ are changed. However, this is not the case, and in the region $\mu$ $>\mu_{c}(H)$ we have infinitely many massless symmetric phases of the theory corresponding to infinitely many Landau levels, as well as a variety of critical curves of secondorder phase transitions. We will now prove this.

It is well known that the state of thermodynamic equilibrium (the ground state) of an arbitrary quantum system is described by the thermodynamic potential (TDP) $\Omega$, which is just the value of the effective potential at its global minimum point. In the case under consideration, the $\operatorname{TDP} \Omega(\mu, H)$ at $\mu>\mu_{c}(H)$ has the form

$$
\begin{aligned}
\Omega(\mu, H) \equiv & V_{H \mu}(0) \\
= & V_{H}(0)-\frac{e H}{4 \pi^{2}} \sum_{k=0}^{\infty} \alpha_{k} \theta\left(\mu-\epsilon_{k}\right) \\
& \times\left\{\mu \sqrt{\mu^{2}-\epsilon_{k}^{2}}-\epsilon_{k}^{2} \ln \left[\left(\sqrt{\mu^{2}-\epsilon_{k}^{2}}+\mu\right) / \epsilon_{k}\right]\right\},
\end{aligned}
$$

where $\epsilon_{k}=\sqrt{2 e H k}$. We shall use the following criterion of phase transitions: if at least one first (second) partial derivative of $\Omega(\mu, H)$ is a discontinuous function at some point, then this is a point of a first- (second-) order phase transition.

Using this criterion, let us show that boundaries of $\omega_{k}$ regions (22), i.e., lines $l_{k}=\{(\mu, H): \mu=\sqrt{2 e H k}\} \quad(k$ $=1,2, \ldots)$, are critical lines of second-order phase transitions. In an arbitrary $\omega_{k}$ region the TDP (25) has the form:

$$
\begin{aligned}
\left.\Omega(\mu, H)\right|_{\omega_{k}} \equiv & \Omega_{k}=V_{H}(0)-\frac{e H}{4 \pi^{2}} \sum_{i=0}^{k} \alpha_{i} \theta\left(\mu-\epsilon_{i}\right) \\
& \times\left(\mu \sqrt{\mu^{2}-\epsilon_{i}^{2}}-\epsilon_{i}^{2} \ln \left[\frac{\left(\sqrt{\mu^{2}-\epsilon_{i}^{2}}+\mu\right)}{\epsilon_{i}}\right]\right) .
\end{aligned}
$$

From Eq. (26) one easily finds

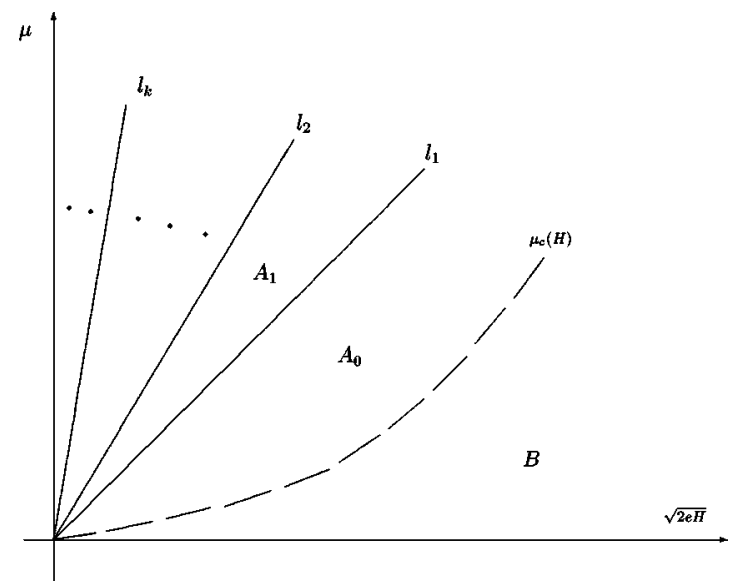

FIG. 3. Phase portrait of the gauged model I at $G<G_{c}$. Solid lines $l_{k}$ are given by $l_{k}=\{(\mu, H): \mu=\sqrt{2 e H k}\}$. They are critical curves of second-order phase transitions. The dashed line of firstorder phase transitions is defined by Eq. (23).

$$
\left.\frac{\partial \Omega_{k}}{\partial \mu}\right|_{(\mu, H) \rightarrow l_{k+}}-\left.\frac{\partial \Omega_{k-1}}{\partial \mu}\right|_{(\mu, H) \rightarrow l_{k-}}=0,
$$

as well as

$$
\begin{array}{r}
\left.\frac{\partial^{2} \Omega_{k}}{(\partial \mu)^{2}}\right|_{(\mu, H) \rightarrow l_{k+}}-\left.\frac{\partial^{2} \Omega_{k-1}}{(\partial \mu)^{2}}\right|_{(\mu, H) \rightarrow l_{k-}} \\
=-\left.\frac{e H \mu}{2 \pi^{2} \sqrt{\mu^{2}-\epsilon_{k}^{2}}}\right|_{\mu \rightarrow \epsilon_{k+}} \rightarrow-\infty .
\end{array}
$$

Equation (27) means that the first derivative $\partial \Omega / \partial \mu$ is a continuous function on all lines $l_{k}$. However, the second derivative $\partial^{2} \Omega /(\partial \mu)^{2}$ has an infinite jump on each line $l_{k}$ [see (28)], so these lines are critical curves of second-order phase transitions. [Similarly, we can prove the discontinuity of $\partial^{2} \Omega /(\partial H)^{2}$ and $\partial^{2} \Omega / \partial \mu \partial H$ on all lines $l_{n}$.]

The results of the above investigations are presented in Fig. 3, where the phase portrait of the NJL model I at $G$ $<G_{c}$ in the $(\mu, H)$ plane is displayed.

\section{The case $G>G_{c}$}

Concerning supercritical values of the coupling constant, we shall consider here only the case $G_{c}<G$ $<(1.225 \ldots) G_{c}$, where the phase portrait of model $\mathrm{I}$ is qualitatively represented in Fig. 4. In this figure one can see infinite sets of symmetric massless $A_{0}, A_{1}, \ldots$ phases, as well as massive phases $C_{0}, C_{1}, \ldots$ with DCSB. In addition, there is another massive phase $B$. Dashed and solid lines in Fig. 4 are critical curves of first- and second-order phase transitions, respectively. One can also see on this portrait infinitely many tricritical points $t_{k}, s_{k}(k=0,1,2, \ldots)$. For a fixed value of $k$ the point $t_{k}$ lies inside, but the point $s_{k}$ is on the left boundary of the corresponding $\omega_{k}$ region (22). Each critical line $l_{k}$ coincides with a part of the $\omega_{k}$ boundary. In 


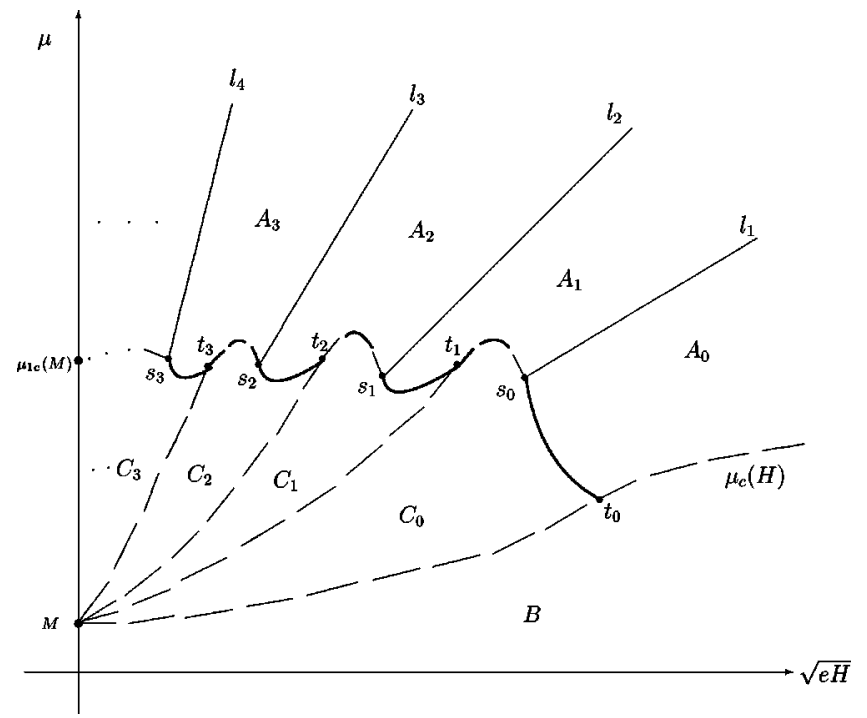

FIG. 4. Phase portrait of the gauged model I at $G_{c}<G$ $<(1.225 \ldots) G_{c}$. Here $M$ is the quark mass at $\mu=0, H=0$, and the quantity $\mu_{1 c}(M)$ is presented in Fig. 1 . The dashed line $\overbrace{t_{0} \mu_{c}(H)}$ is defined by Eq. (23). In this case one has infinite sets of symmetric massless phases $A_{0}, A_{1}, \ldots$ as well as massive phases $C_{0}, C_{1}, \ldots$ with DCSB. In addition there exists another massive phase $B$.

Table I, we give the values of the external magnetic field corresponding to tricritical points $t_{0}$ and $s_{0}$.

The presence of an infinite cascade of massless $A_{k}$ phases in the case $G>G_{c}$ may be proved in a way similar to what was done in the previous subsection. However, now an infinite set of massive chirally nonsymmetric phases is available thanks to the particular structure of the function $\phi(\Sigma)$ in Eq. (21). A detailed numerical investigation of this function shows that, for some values of $(\mu, H)$ inside the $C_{k}$ region (see Fig. 4), $\phi(\Sigma)$ as a function of $\Sigma$ qualitatively behaves like the curve, drawn in Fig. 5. At these values of $(\mu, H)$ there is only one nontrivial solution $\Sigma_{k}(\mu, H)$ of the stationarity Eq. (21), which is the global minimum point of the effective potential (20) and at the same time is the quark mass in the phase $C_{k}$ of the theory. Remark that in each phase $C_{k}$ the quark mass is a $\mu$-dependent function. In contrast, in the phase $B$, the global minimum point is equal to $\Sigma_{0}(H)$ [see Eqs. (16) and (17)], which is a $\mu$-independent quantity. Hence, the particle density $n \equiv-\partial \Omega / \partial \mu$ in the ground state of phase $B$ is identically equal to zero, whereas in each phase $C_{k}$ this quantity differs from zero. This conclusion follows from the definition of the thermodynamic potential $\Omega$ given in the previous subsection, as well as from

TABLE I. Values of the external magnetic field corresponding to tricritical points $t_{0}$ and $s_{0}$ (see Fig. 4) for different ratios of coupling constants $G / G_{c}$.

\begin{tabular}{lcccc}
\hline \hline$G / G_{c}$ & 1.01 & 1.1 & 1.15 & 1.2 \\
\hline$e H_{t_{0}} / \Lambda^{2}$ & $0.0129 \ldots$ & $0.08119 \ldots$ & $0.10769 \ldots$ & $0.12987 \ldots$ \\
$e H_{s_{0}} / \Lambda^{2}$ & $0.00614 \ldots$ & $0.05639 \ldots$ & $0.08088 \ldots$ & $0.10338 \ldots$ \\
\hline \hline
\end{tabular}

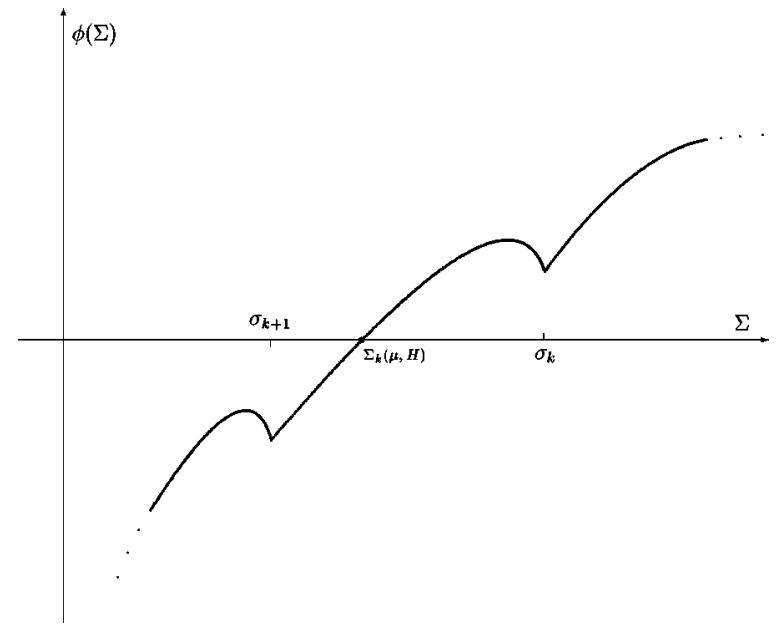

FIG. 5. Typical behavior of $\phi(\Sigma)$ (21) for some points $(\mu, H)$ $\in C_{k}$. Here $\sigma_{n}=\sqrt{\mu^{2}-2 e H n}$.

the fact that $\Omega$ has no $\mu$ dependence in the phase $B .^{4}$

If $\mu$ increases, the curve of Fig. 5 moves up and to the right-hand side of this figure. So, for some values of $\mu$ the function $\phi(\Sigma)$ in Eq. (21) will have three zeros (see Fig. 6): the one on the left-hand side in this figure, $\Sigma_{k+1}$, is a local minimum of the function $V_{H \mu}(\Sigma)$, the one on the right-hand side, $\Sigma_{k}\left(\Sigma_{k}>\Sigma_{k+1}\right)$, a global minimum of that function. If the chemical potential persists to grow, then at some critical value of $\mu$ the global minimum jumps from $\Sigma_{k}(\mu, H)$ to $\Sigma_{k+1}(\mu, H)$. At this moment we have a first-order phase transition from the massive phase $C_{k}$ to the massive $C_{k+1}$ one. In Fig. 4 the point of this phase transition lies on the curve

$$
\overbrace{M t_{k+1}},
$$

which is the boundary between regions $C_{k}$ and $C_{k+1}$. Hence, all lines

$$
\overbrace{M t_{k}}
$$

in Fig. $4(k=0,1,2, \ldots)$ are first-order phase-transition curves. Here we should also remark that all points of the line

$$
\overbrace{t_{0} \mu_{c}(H)}
$$

in this figure are described by Eq. (23). Since the phase structure of model I is so complicated, the dynamical quark mass $\Sigma(\mu, H)$, which is given by the global minimum of the potential $V_{H \mu}(\Sigma)$, also has a rather complicated $\mu, H$ dependence. For illustration, in Fig. 7 the schematic behavior of $\Sigma(\mu, H)$ versus $\mu$ is presented at some fixed value of the external magnetic field $H$.

From standard textbooks on statistical physics (see, e.g., [4]) we know that more than three curves of first-order phase transitions (1OPT) should not intersect at one point of a phase diagram; thus, not more than three phases are allowed

\footnotetext{
${ }^{4}$ Since for all points of region $B$ we have $\mu<\Sigma_{0}(H)$, it follows from Eq. (20) that $\Omega=V_{H \mu}\left(\Sigma_{0}(H)\right)=V_{H}\left(\Sigma_{0}(H)\right)$, i.e., $\Omega$ has indeed no $\mu$ dependence.
} 


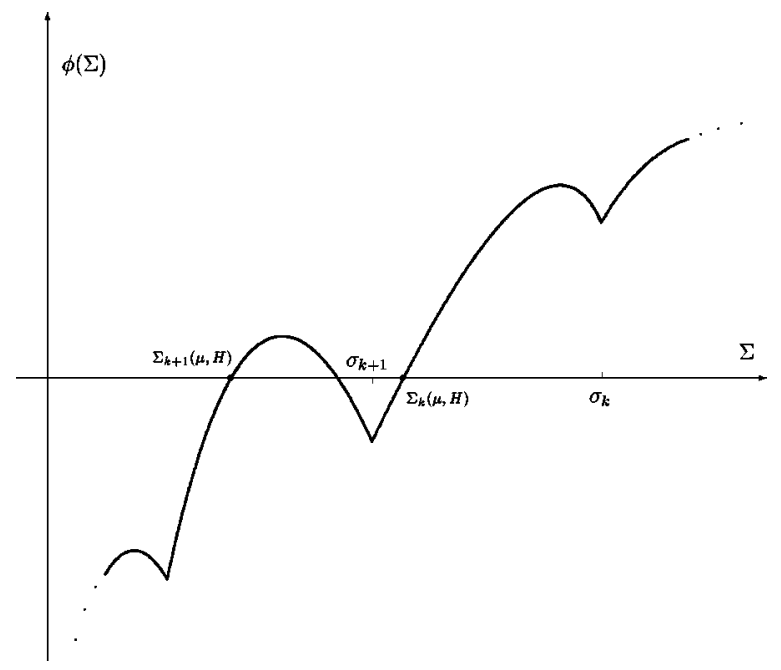

FIG. 6. For increasing $\mu$, there arise three zeroes of the function $\phi(\Sigma)$ (21) defining two local minima $\Sigma_{k}$ and $\Sigma_{k+1}\left(\Sigma_{k+1}<\Sigma_{k}\right)$ of the effective potential, respectively. The global minimum of $V_{H \mu}(\Sigma)$ lies in one of them and passes by a jump from one local minimum to another one depending on the values of $\mu$.

to coexist in nature. However, in Fig. 4 one can see that an infinite number of curves of 1OPT cross at the one point $M$. Indeed, this visible contradiction with the above-mentioned statement is only fictitious, because at the point $M$ we have a second-order phase transition [14]. Hence, $M$ does not belong to any of the critical curves of 1OPT

$$
\overbrace{M t_{k}}
$$

and is therefore not a point of phase coexistence.

\section{MAGNETIC OSCILLATIONS IN MODEL I}

Now we want to show that there arise, from the presence of infinite sets of massless $A_{k}$ phases as well as of massive $C_{k}$ ones, magnetic oscillations (the so-called van Alphen-de Haas-type effect) of some physical parameters in model I gauged by an external magnetic field.

\section{A. The case $G<G_{c}$}

Let the chemical potential be fixed, i.e., $\mu=$ const $>\mu_{c}(H)$. Then on the plane $(\mu, H)$ (see Fig. 3) we have a line that crosses critical lines $l_{1}, l_{2}, \ldots$ at points $H_{1}, H_{2}, \ldots$. The particle density $n$ and the magnetization $m$ of any thermodynamic system are defined by the TDP in the following way: $n=-\partial \Omega / \partial \mu, m=-\partial \Omega / \partial H$. At $\mu=$ const these quantities are continuous functions of the external magnetic field only, i.e., $n \equiv n(H), m \equiv m(H)$. We know that all the second derivatives of $\Omega(\mu, H)$ are discontinuous on every critical line $l_{n}$. The functions $n(H)$ and $m(H)$, being continuous in the interval $H \in(0, \infty)$, therefore have first derivatives that are discontinuous on an infinite set of points $H_{1}, \ldots, H_{k}, \ldots$. Such a behavior manifests itself as a phenomenon usually called oscillation.

In condensed-matter physics $[3,4]$ it is a conventional rule to separate the expression for a physical quantity with oscil-

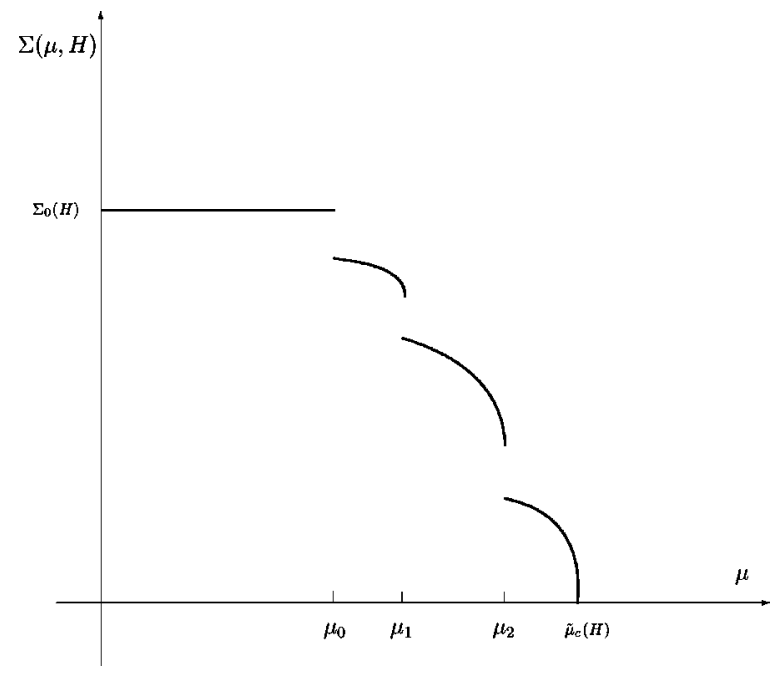

FIG. 7. Schematic representation of the dynamical quark mass $\Sigma(\mu, H)$ as a function of $\mu$ for the fixed magnetic field qualitatively reflecting the structure of the phase portrait in Fig. 4. Here the magnetic field is fixed in the interval $\left(H_{s_{2}}, H_{t_{2}}\right)$, where $s_{2}, t_{2}$ are tricritical points (see Fig. 4). The $\mu_{k}, \tilde{\mu}_{c}(H)(k=0,1,2)$ are the values of the chemical potential at which the line $H=$ const crosses in Fig. 4 the critical curves $\overbrace{M t_{k}}$ and $\overbrace{s_{2} t_{2}}$, respectively.

lations into two parts; the first one is called monotonic and does not contain any oscillations, whereas the second part, which is of particular interest here, contains all the oscillations. Following this rule, we can write down, say, the TDP (25) of the NJL model I in the form

$$
\Omega(\mu, H)=\Omega_{m o n}(\mu, H)+\Omega_{o s c}(\mu, H) .
$$

In order to present the oscillating part $\Omega_{\text {osc }}(\mu, H)$ as well as the monotonic one $\Omega_{\text {mon }}(\mu, H)$ in an analytical form, we shall use the technique elaborated in [6], where manifestly analytical expressions for these quantities were found in the case of a perfectly relativistic electron-positron gas. This technique can be used without any difficulties in our case, too. So, by applying the Poisson summation formula [4] in Eq. (25),

$$
\sum_{n=0}^{\infty} \alpha_{n} \Phi(n)=2 \sum_{k=0}^{\infty} \alpha_{k} \int_{0}^{\infty} \Phi(x) \cos (2 \pi k x) d x
$$

one can get for $\Omega_{m o n}(\mu, H)$ and $\Omega_{o s c}(\mu, H)$ the following expressions:

$$
\begin{aligned}
\Omega_{m o n}= & V_{H}(0)-\frac{\mu^{4}}{12 \pi^{2}}-\frac{(e H)^{2}}{4 \pi^{3}} \int_{0}^{\nu} d y \sum_{k=1}^{\infty} \frac{1}{k} P(\pi k y), \\
\Omega_{o s c}= & \frac{\mu}{4 \pi^{3 / 2}} \sum_{k=1}^{\infty}\left(\frac{e H}{\pi k}\right)^{3 / 2}[Q(\pi k \nu) \cos (\pi k \nu+\pi / 4) \\
& +P(\pi k \nu) \cos (\pi k \nu-\pi / 4)],
\end{aligned}
$$


where $\nu=\mu^{2} /(e H)$. [To find Eqs. (31) and (32) it is sufficient to let the electron mass pass to zero in formula (19) of [6].] Functions $P(x)$ and $Q(x)$ in Eqs. (31) and (32) are connected with Fresnel integrals $C(x)$ and $S(x)$ [33]

$$
\begin{aligned}
& C(x)=\frac{1}{2}+\sqrt{\frac{x}{2 \pi}}[P(x) \sin x+Q(x) \cos x] \\
& S(x)=\frac{1}{2}-\sqrt{\frac{x}{2 \pi}}[P(x) \cos x-Q(x) \sin x] .
\end{aligned}
$$

They have, at $x \rightarrow \infty$, the following asymptotics [33]:

$$
P(x)=x^{-1}-\frac{3}{4} x^{-3}+\cdots, \quad Q(x)=-\frac{1}{2} x^{-2}+\frac{15}{8} x^{-4}+\cdots .
$$

Formula (32) presents, in a manifestly analytical form, the oscillating part of the TDP (25) for the NJL model at $G$ $<G_{c}$. In the case under consideration, since the TDP is proportional to the pressure of the system, one can conclude that the pressure in the NJL model oscillates when $H \rightarrow 0$, too. It follows from Eq. (32) that the frequency of oscillations over the parameter $(e H)^{-1}$ equals $\mu^{2} / 2$. Then, starting from Eq. (32), one can easily find the corresponding expressions for the oscillating parts of $n(H)$ and $m(H)$. These quantities oscillate at $H \rightarrow 0$ with the same frequency $\mu^{2} / 2$ and have a rather involved form, so we do not present them here.

Finally, we should note that the character of magnetic oscillations in the NJL model at $G<G_{c}$ resembles the magnetic oscillations in massless quantum electrodynamics $[5,6]$. This circumstance is conditioned by the resemblance of the vacuum properties in the two models. Indeed, both in the NJL model and in QED, for fixed $\mu$ and varying values of $H$, there are infinitely many second-order phase transitions (see the Appendix, where the vacuum structure of QED at $\mu, H$ $\neq 0$ is considered).

\section{B. The case $G_{c}<G<(1.225 \ldots) G_{c}$}

Here at $\mu>\mu_{1 c}(M)$ (see Fig. 4) the TDP of the NJL model as well as all thermodynamical parameters of the system oscillate with the frequency $\mu^{2} / 2$. This can be shown in a way similar to what was done in the previous section.

However, at $M<\mu<\mu_{1 c}(M)$, the character of magnetic oscillations is changed. We will prove this next. First of all, in this case we have another expression for the TDP of the system,

$$
\Omega(\mu, H)=V_{H \mu}(\Sigma(\mu, H)),
$$

where $V_{H \mu}(\Sigma)$ is given in Eq. (20) and $\Sigma(\mu, H)$ is the nontrivial solution of the stationarity equation (21). In each of the massive phases $C_{k}$ the $\mu$ - and $H$-dependent function $\Sigma(\mu, H)$ coincides with the corresponding fermionic mass $\Sigma_{k}(\mu, H)$ (see Sec. III C). By using the Poisson summation formula (30) again in Eq. (20), one can easily select the oscillating part of the TDP (33) in a manifestly analytical form

$$
\begin{aligned}
\Omega_{o s c}(\mu, H)= & \frac{\mu \theta(\mu-\Sigma(\mu, H))}{4 \pi^{3 / 2}} \sum_{k=1}^{\infty}\left(\frac{e H}{\pi k}\right)^{3 / 2} \\
& \times[Q(\pi k \nu) \cos (2 \pi k \omega+\pi / 4) \\
& +P(\pi k \nu) \cos (2 \pi k \omega-\pi / 4)],
\end{aligned}
$$

where $\nu=\mu^{2} /(e H), \omega=\left[\mu^{2}-\Sigma^{2}(\mu, H)\right] /(2 e H)$. From Eq. (34) one can see that as a function of the variable $(e H)^{-1}$, the TDP (33) oscillates with frequency $\left[\mu^{2}-\Sigma^{2}(\mu, H)\right] / 2$ if this variable tends to infinity. Since $\Omega(\mu, H)$ is equal, up to a sign, to the pressure in the ground state of the system, the pressure in the NJL model in the present case is also an oscillating quantity. Moreover, taking into account the stationarity Eq. (21), one can easily derive manifest expressions for oscillating parts of other thermodynamic quantities such as particle density $n=-\partial \Omega / \partial \mu$ and magnetization $m=$ $-\partial \Omega / \partial H$ from Eqs. (33) and (34),

$$
\begin{aligned}
m_{\text {osc }}= & -\frac{\left[\mu^{2}-\Sigma^{2}(\mu, H)\right]^{3 / 2}}{4 \sqrt{2} \pi^{3} \mu \omega^{1 / 2}} \\
& \times \sum_{k=1}^{\infty} \frac{\sin (2 \pi k \omega-\pi / 4)}{k^{3 / 2}}+o\left(\frac{1}{\omega^{1 / 2}}\right), \\
n_{\text {osc }}= & \frac{\left[\mu^{2}-\Sigma^{2}(\mu, H)\right]^{3 / 2}}{4 \sqrt{2} \pi^{3} \mu \omega^{3 / 2}} \sum_{k=1}^{\infty} \frac{\sin (2 \pi k \omega-\pi / 4)}{k^{3 / 2}} .
\end{aligned}
$$

It is clear from Eq. (35) that particle density and magnetization in the ground state of the NJL model oscillate with the same frequency as $\Omega$.

Comparing magnetic oscillations in the NJL model I and in QED, we see three main differences. Let us remark that in QED the frequency of magnetic oscillations [over the variable $(e H)^{-1}$ ] is equal to $\left(\mu^{2}-M^{2}\right) / 2$, where $M$ is the electron mass [6]. In the NJL model, in contrast to QED, the magnetic oscillation frequency is an $H$-dependent quantity. So, strictly speaking, in the NJL model magnetic oscillations are not periodic ones. Similar peculiarities of magnetic oscillations are observed in some ferromagnetic semiconductive materials such as $\mathrm{HgCr}_{2} \mathrm{Se}_{4}$ [34], where nonperiodic magnetic oscillations over the variable $(e H)^{-1}$ were found to exist for electric conductivity ${ }^{5}$ as well as magnetization. This is the first distinction.

A second difference is the character of the oscillations in the two models: in QED, magnetic oscillations are accompanied by second-order phase transitions (see the Appendix), while in the NJL model they occur as a result of an infinite cascade of first-order phase transitions.

Third, we should remark that in the NJL model not only thermodynamic quantities oscillate, but some dynamical parameters of the system do as well. This concerns, in particu-

\footnotetext{
${ }^{5}$ Magnetic oscillations of the electric conductivity, which is proportional to the particle density, are known as the Shubnikov-de Haas effect [3].
} 
lar, oscillations of the dynamical quark mass. In fact, by applying the Poisson summation formula (30) in the stationarity Eq. (21) and searching for the solution $\Sigma(\mu, H)$ of this equation in the form $\Sigma(\mu, H)=\Sigma_{m o n}+\Sigma_{o s c}$, one can easily find the following expressions for $H \rightarrow 0$ :

$$
\begin{aligned}
& \Sigma_{o s c}=\frac{\left(\mu^{2}-\tilde{M}^{2}\right)^{3 / 2}}{\sqrt{2} \pi \mu \tilde{\omega}^{3 / 2} f^{\prime}(\tilde{M})} \sum_{k=1}^{\infty} \frac{\sin (2 \pi k \tilde{\omega}-\pi / 4)}{k^{3 / 2}}+o\left(\frac{1}{\omega^{3 / 2}}\right), \\
& \Sigma_{\text {mon }}=\tilde{M}+\frac{\mu\left(\mu^{2}-\tilde{M}^{2}\right)^{3 / 2}}{12 \tilde{M}^{2} f^{\prime}(\tilde{M}) \tilde{\omega}^{2}}\left[1+\frac{\sqrt{\mu^{2}-\tilde{M}^{2}}}{\mu}\right]+o\left(\frac{1}{\omega^{3 / 2}}\right),
\end{aligned}
$$

where $\tilde{\omega}=\left(\mu^{2}-\tilde{M}^{2}\right) / 2(e H), \quad \widetilde{M} \equiv \Sigma_{0}(\mu)$ is the quark mass at $H=0, \quad \mu \neq 0$ (see Fig. 2) and

$$
f(x)=F(x)+2 \mu \sqrt{\mu^{2}-x^{2}}-2 x^{2} \ln \frac{\mu+\sqrt{\mu^{2}-x^{2}}}{x}
$$

$[F(\Sigma)$ is defined in (7)]. Hence, in the framework of the NJL model I the quark mass $\Sigma(\mu, H)$, as well as other dynamical quantities composed from it, oscillate in the presence of an external magnetic field.

\section{MAGNETIC OSCILLATIONS IN MODEL II}

Next, let us consider magnetic oscillations in the more realistic NJL model II containing two kinds of quarks: $u$ and $d$ quarks with electric charges $e_{1}$ and $e_{2}$, respectively. The effective potential $\mathrm{v}_{H \mu}$ in the case under consideration is a trivial generalization of $V_{H \mu}$ derived in the one-flavor case

$$
\mathrm{v}_{H \mu}(\Sigma)=-\frac{H^{2}}{2}-\frac{\Sigma^{2}}{2 G}+\sum_{i=1}^{2} V_{e_{i} H \mu}(\Sigma)
$$

where $\Sigma=\sqrt{\sigma^{2}+\vec{\pi}^{2}}$, and $V_{e_{i} H \mu}(\Sigma)$ is equal to $V_{H \mu}(\Sigma)(20)$, with $e$ replaced by $\left|e_{i}\right|$.

Qualitatively, the phase structure of the NJL model II is the same as that of model I. So, at $G<G_{c}$ we have an infinite set of massless phases (similar to the phase portrait of model I in Fig. 3) reflecting the infinite set of Landau levels that is the basis for magnetic oscillations. Using the analytical methods of Sec. IV, one can easily select in the present case the oscillating part of the thermodynamic potential:

$$
\begin{aligned}
\Omega_{o s c}= & \frac{\mu}{4 \pi^{3 / 2}} \sum_{i=1}^{2} \sum_{k=1}^{\infty}\left(\frac{\left|e_{i}\right| H}{\pi k}\right)^{3 / 2} \\
& \times\left[Q\left(\pi k \nu_{i}\right) \cos \left(\pi k \nu_{i}+\pi / 4\right)\right. \\
& \left.+P\left(\pi k \nu_{i}\right) \cos \left(\pi k \nu_{i}-\pi / 4\right)\right],
\end{aligned}
$$

where $\nu_{i}=\mu^{2} /\left(\left|e_{i}\right| H\right)$. Hence, in model II, in contrast to model I and QED, we have a superposition of two oscillating modes. At growing values of the parameter $(e)^{-1}$, the frequency of oscillations in each of the modes is equal to $e \mu^{2} /\left(2\left|e_{i}\right|\right)$.
At $G_{c}<G$ there is a finite vicinity of $G_{c}$ in which the phase portrait of model II in the $(\mu, H)$ plane is similar to the one in model I (see Fig. 4). So, in the case under consideration we have infinite sets of massless and massive phases as well. The cascade of massive phases is the foundation for nonperiodic magnetic oscillations. Indeed, from Eq. (37) it follows that the oscillating part of TDP has the form

$$
\begin{aligned}
\Omega_{o s c}(\mu, H)= & \frac{\mu \theta(\mu-\Sigma(\mu, H))}{4 \pi^{3 / 2}} \sum_{i=1}^{2} \sum_{k=1}^{\infty}\left(\frac{\left|e_{i}\right| H}{\pi k}\right)^{3 / 2} \\
& \times\left[Q\left(\pi k \nu_{i}\right) \cos \left(2 \pi k \omega_{i}+\pi / 4\right)\right. \\
& \left.+P\left(\pi k \nu_{i}\right) \cos \left(2 \pi k \omega_{i}-\pi / 4\right)\right],
\end{aligned}
$$

where $\nu_{i}=\mu^{2} /\left(\left|e_{i}\right| H\right), \quad \omega_{i}=\left[\mu^{2}-\Sigma^{2}(\mu, H)\right] /\left(2\left|e_{i}\right| H\right)$, and $\Sigma(\mu, H)$ is the global minimum point of the effective potential (37). As in the previous model, $\Sigma(\mu, H)$ in the present case is an $H$-dependent function. This means that magnetic oscillations in the NJL model II are composed of two nonperiodic harmonics, because each of them has, as a function of the variable $(e H)^{-1}$, the $H$-dependent frequency $e\left[\mu^{2}\right.$ $\left.-\Sigma^{2}(\mu, H)\right] /\left(2\left|e_{i}\right|\right)$.

\section{SUMMARY AND CONCLUSIONS}

In the present paper we have studied the magnetic properties of a many-body system of cold and dense quark matter with four-fermion interactions. In particular, we have investigated the ground-state (vacuum) structure of two simple NJL models with one or two quark flavors, respectively, which are taken at nonzero chemical potential $\mu$ and magnetic field $H$.

As it turns out, in both types of models there exists a phase $B$ (see Figs. 3 and 4 ) in which the quark mass is equal to $\Sigma_{0}(H)$, i.e., it is a $\mu$-independent quantity. Since this phase is achieved in the region $\mu<\Sigma_{0}(H)$, the resulting particle density is expected to be zero, which is supported by our calculation. Clearly, this is in agreement with the physical interpretation of the chemical potential as the energy required to create one particle in the system. Indeed, the energy $\mu$, which in the phase $B$ is smaller than the quark mass, is not sufficient to create a particle, so that in the ground state of this phase the particle density must vanish.

Most interestingly, we have shown that in NJL models there exist an infinite set of massless chirally invariant phases (phases $A_{0}, A_{1}, \ldots$ in Figs. 3 and 4), which lead to periodic magnetic oscillations of some thermodynamic quantities of the system (so-called van Alphen-de Haas effect). In NJL models, this effect is observed at weak couplings $\left(G<G_{c}\right.$, where $\left.G_{c}=4 \pi^{2} / \Lambda^{2}\right)$ or at sufficiently high values of the chemical potential and resembles magnetic oscillations in massless QED.

Furthermore, for some finite interval of the coupling constant $G_{c}<G<G_{1}$, where in the framework of model I $G_{1}$ $=(1.225 \ldots) G_{c}$, the phase structure of NJL models I and II contains an infinite set of massive chirally noninvariant phases (phases $C_{0}, C_{1}, \ldots$ in Fig. 4). This is the basis for nonperiodic magnetic oscillations of some thermodynamic 
parameters, since the dynamical quark mass in each of the phases $C_{k}$ is now itself an $H$-dependent quantity. (Notice that analogous nonperiodic magnetic oscillations were recently found to exist in some condensed-matter materials [34], too.) We should also remark that, at $G_{c}<G<G_{1}$, some dynamical parameters in NJL systems, such as quark masses, oscillate over $(e H)^{-1}$ as well. This is an unknown fact in the standard condensed-matter theory of the van Alphen-de Haas effect.

Moreover, our numerical analysis shows that for values of the coupling constant $G \in\left(G_{1}, G_{2}\right)$, where $G_{2} \approx 40 G_{c}$, we have in both models a finite number of massive phases $C_{k}$ (the number of massless phases is infinite as before). At larger values of the coupling constant, i.e., at $G>G_{2}$, there exist no massive phases in the phase structure of NJL models at all, except the trivial phase $B$.

It is further interesting to note that for fixed magnetic field $H$ the dynamical quark mass $\Sigma(\mu, H)$ of NJL models discontinuously jumps as a function of $\mu$, at points $\mu_{0}, \mu_{1}, \mu_{2}, \ldots$ (see Fig. 7), thus reflecting the structure of the underlying phase portrait shown in Fig. 4.

In conclusion, we have shown that NJL models at $\mu, H$ $\neq 0$ exhibit an interesting phase structure and a set of oscillating quantities, which are richer than in the corresponding QED case. Finally, it is worth mentioning that extended NJL models, which allow for the generation of a nonvanishing $\langle q q\rangle$-condensate for larger values of the chemical potential, present the exciting new possibility of a spontaneous breaking of color $\mathrm{SU}(3)$ and electromagnetic U(1) symmetry with the arising Meissner effects and with the emergence of a new modified unbroken $\mathrm{U}(1)$ symmetry. Note that our approach can be employed for a combined study of quark and diquark condensates of extended NJL models taken at $\mu, H \neq 0$. Work in this direction is under way.

\section{ACKNOWLEDGMENTS}

One of the authors (D.E.) gratefully acknowledges the kind support and warm hospitality of the colleagues of the Theory Division at CERN. This work was supported in part by the Russian Fund for Fundamental Research, project 9802-16690, and by DFG-project 436 RUS 113/477.

\section{APPENDIX: THE QED VACUUM STRUCTURE IN THE PRESENCE OF $\mu$ AND $H$.}

In the framework of QED and in a one-loop approximation, the effective Lagrangian in the presence of an external homogeneous magnetic field has the following form $[5,6]$ :

$$
L_{e f f}(\mu, H)=L_{1}(H)-\Omega_{Q E D}(\mu, H),
$$

where $L_{1}(H)$ is the Lagrangian at $\mu=0, H \neq 0$. Since it does not influence the phase structure of QED, we do not present its explicit form here, but refer to $[5,6]$. The second part of $L_{e f f}(\mu, H)$ is exactly the thermodynamic potential of a perfect electron-positron gas

$$
\begin{aligned}
\Omega_{Q E D}(\mu, H)= & -\frac{e H}{4 \pi^{2}} \sum_{n=0}^{\infty} \alpha_{n} \theta\left(\mu-\epsilon_{n}\right) \\
& \times\left\{\mu \sqrt{\mu^{2}-\epsilon_{n}^{2}}-\epsilon_{n}^{2} \ln \left[\left(\sqrt{\mu^{2}-\epsilon_{n}^{2}}+\mu\right) / \epsilon_{n}\right]\right\} .
\end{aligned}
$$

In (A2) we have introduced the notation $\epsilon_{n}=\sqrt{M^{2}+2 e H n}$, where $M$ is the electron mass at $H=0, \mu=0$, and $\alpha_{n}=2$ $-\delta_{0 n}$.

Using the results and methods of Sec. III B one can now easily show that, on each line $l_{n}=\{(\mu, H): \mu$ $\left.=\sqrt{M^{2}+2 e H n}\right\}$ of the $(\mu, H)$ plane, all second derivatives of $L_{e f f}(\mu, H)$ (A1) are discontinuous. So, the lines $l_{n}(n$ $=0,1, \ldots)$ in Fig. 8 are the curves of second-order phase transitions. They divide the $(\mu, H)$ plane into an infinite set of regions $C_{n} \quad(n=0,1, \ldots)$ corresponding to different massive phases of QED (associated with Landau levels) with the same electron mass $M$. Nevertheless, each phase $C_{n}$ is characterized by such physical quantities as particle density $n(\mu, H)$ and magnetization $m(\mu, H)$. On each phase boundary $l_{n}$ these quantities are continuous functions. However, their first derivatives are discontinuous functions on each line $l_{n}$, so the derivative jump of $n$ or $m$ is the signal of a second-order phase transition.

As in the previously considered case (see Sec. IIIC and Fig. 4) the point $M$ in Fig. 8, where all lines $l_{n}$ intersect, is a special point differing from other points of the lines $l_{n}$. Indeed, at $H=0$ we have [6]

$$
\Omega_{Q E D}(\mu, 0)=-\frac{\theta(\mu-M)}{6 \pi^{2}} \int_{M}^{\mu} \frac{x d x}{\sqrt{x^{2}-M^{2}}}(\mu-x)^{2}(\mu+2 x) .
$$

It follows from (A3) that only the third derivative

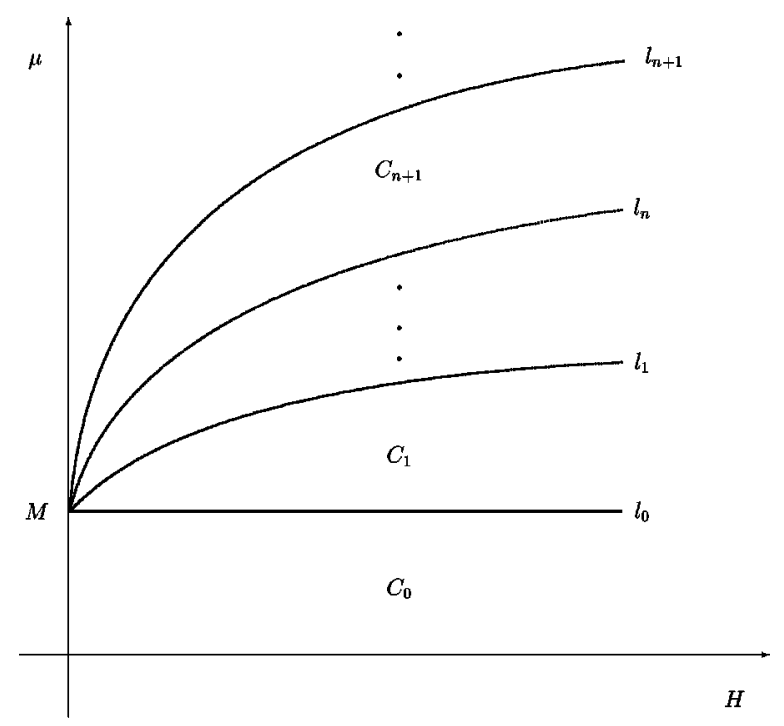

FIG. 8. Phase portrait of QED at $\mu, H \neq 0$. All the lines $l_{n}$ are the curves of second-order phase transitions. 
$\partial^{3} \Omega_{Q E D}(\mu, 0) /(\partial \mu)^{3}$ is a discontinuous function at the point $\mu=M$. At the same time, in other points of critical curves $l_{n}$, already the second derivative $\partial^{2} \Omega_{Q E D}(\mu, H) /(\partial \mu)^{2}$ is discontinuous.

Here we have considered only the relativistic case, but one can easily show that the nonrelativistic electron gas at $\mu, H \neq 0$ has an infinite cascade of massive phases, too. So, at the basis of the van Alphen-de Haas and Shubnikov-de Haas effects lies an infinite set of second-order phase transitions.
[1] D. Bailin and A. Love, Phys. Rep. 107, 325 (1984); R. Rapp, T. Schafer, E.V. Shuryak, and M. Velkovsky, Phys. Rev. Lett. 81, 53 (1998); M. Alford, K. Rajagopal, and F. Wilczek, Phys. Lett. B 422, 247 (1998).

[2] S. Schramm, B. Muller, and A.J. Schramm, Mod. Phys. Lett. A 7, 973 (1992); I.A. Shushpanov and A.V. Smilga, Phys. Lett. B 402, 351 (1997); J. Rafelsky, Electromagnetic Fields in the QCD-Vacuum, hep-ph/9806389.

[3] W.J. de Haas and P.M. van Alphen, Proc. Am. Acad. Arts Sci. 33, 1106 (1936); D. Shoenberg, Magnetic Oscillations in Metals (Cambridge University Press, Cambridge, England, 1984); I.M. Lifshitz, Selected Works. Electronic Theory of Metals, Physics of Polymers and Bipolymers (Nauka, Moscow, 1994) (in Russian).

[4] E.M. Lifshitz and L.P. Pitaevski, Statistical Physics (Pergamon, Oxford, 1980); Yu.B. Rumer and M.Sh. Ryvkin, Thermodynamics, Statistical Physics and Kinetics (Nauka, Moscow, 1977) (in Russian).

[5] P. Elmfors, D. Persson, and B.-S. Skagerstam, Phys. Rev. Lett. 71, 480 (1993); Astropart. Phys. 2, 299 (1994); D. Persson and V. Zeitlin, Phys. Rev. D 51, 2026 (1995); J.O. Andersen and T. Haugset, ibid. 51, 3073 (1995); V.Ch. Zhukovsky, T.L. Shoniya, and P.A. Eminov, Zh. Eksp. Teor. Fiz. 107, 299 (1995) [JETP 80, 158 (1995)]; V.Ch. Zhukovsky, A.S. Vshivtsev, and P.A. Eminov, Yad. Fiz. 58, 1274 (1995) [Phys. At. Nucl. 58, 1195 (1995)]; V.R. Khalilov, ibid. 61, 1631 (1998) [61, 1520 (1998)].

[6] A.S. Vshivtsev and K.G. Klimenko, Zh. Eksp. Teor. Fiz. 109, 517 (1996) [JETP 82, 514 (1996)].

[7] S. Chakrabarty, Phys. Rev. D 54, 1306 (1996).

[8] Y. Nambu and G. Jona-Lasinio, Phys. Rev. 122, 345 (1961); 124, 246 (1961).

[9] V.G. Vaks and A.I. Larkin, Zh. Eksp. Teor. Fiz 40, 282 (1961) [Sov. Phys. JETP 13, 192 (1961)]; 40, 1392 (1961) [13, 979 (1961]; B.A. Arbuzov, A.N. Tavkhelidze, and R.N. Faustov, Dokl. Akad. Nauk. SSSR 139, 345 (1962) [Sov. Phys. Dokl. 6, 598 (1962)].

[10] D. Ebert and M. K. Volkov, Yad. Fiz. 26, 1265 (1982) [Sov. J. Nucl. Phys. 26, 668 (1982)]; Z. Phys. C 16, 205 (1983); D. Ebert and H. Reinhardt, Nucl. Phys. B271, 188 (1986).

[11] M.K. Volkov, Ann. Phys. (N.Y.) 157, 282 (1984); D. Ebert, H. Reinhardt, and M.K. Volkov, Prog. Part. Nucl. Phys. 33, 1 (1994).

[12] S.P. Klevansky, Rev. Mod. Phys. 64, 649 (1992).

[13] S. Kawati and H. Miyata, Phys. Rev. D 23, 3010 (1981); J. Fuchs, Z. Phys. C 22, 83 (1984); V. Bernard, U.-G. Meissner, and I. Zahed, Phys. Rev. D 36, 819 (1987); Chr.V. Christov and K. Goeke, Acta Phys. Pol. B 22, 187 (1991); D. Ebert, Yu.L. Kalinovsky, L. Münchow, and M.K. Volkov, Int. J. Mod. Phys. A 8, 1295 (1993).
[14] A.S. Vshivtsev and K.G. Klimenko, Pis'ma Zh. Eksp. Teor. Fiz. 64, 344 (1996) [JETP Lett. 64, 338 (1996)]; A.S. Vshivtsev, V.Ch. Zhukovsky, and K.G. Klimenko, Zh. Eksp. Teor. Fiz. 111, 1921 (1997) [JETP 84, 1047 (1997)].

[15] S.P. Klevansky and R.H. Lemmer, Phys. Rev. D 39, 3478 (1989).

[16] D. Ebert and M.K. Volkov, Phys. Lett. B 272, 86 (1991); I.A. Shovkovy and V.M. Turkowski, ibid. 367, 213 (1995); D. Ebert and V.Ch. Zhukovsky, Mod. Phys. Lett. A 12, 2567 (1997); A.Yu. Babansky, E.V. Gorbar, and G.V. Shchepanyuk, Phys. Lett. B 419, 272 (1998).

[17] V.P. Gusynin, V.A. Miransky, and I.A. Shovkovy, Phys. Lett. B 349, 477 (1995).

[18] T. Inagaki, T. Muta, and S.D. Odintsov, Mod. Phys. Lett. A 8, 2117 (1993); E. Elizalde, S. Leseduarte, and S.D. Odintsov, Phys. Rev. D 49, 5551 (1994); H. Forkel, Phys. Lett. B 280, 5 (1992); Nucl. Phys. A581, 557 (1995); D.K. Kim and I.G. Koh, Phys. Rev. D 51, 4573 (1995); E.J. Ferrer, V.P. Gusynin, and V. de la Incera, Phys. Lett. B 455, 217 (1999); E.V. Gorbar, hep-th/9904180.

[19] A.S. Vshivtsev, A.K. Klimenko, and K.G. Klimenko, Yad. Fiz. 61, 543 (1998) [Phys. At. Nucl. 61, 479 (1998)]; M.A. Vdovichenko, A.S. Vshivtsev, and K.G. Klimenko, IFVE 97-59, Protvino, 1997 [in Russian]; A.S. Vshivtsev, M.A. Vdovichenko, and K.G. Klimenko, Zh. Eksp. Teor. Fiz. 114, 418 (1998) [JETP 87, 229 (1998)].

[20] T. Inagaki, T. Muta, and S.D. Odintsov, Prog. Theor. Phys. Suppl. 127, 93 (1997).

[21] D.M. Gitman, S.D. Odintsov, and Yu.I. Shil'nov, Phys. Rev. D 54, 2968 (1996); B. Geyer, L.N. Granda, and S.D. Odintsov, Mod. Phys. Lett. A 11, 2053 (1996); E. Elizalde, Yu.I. Shil'nov, and V.V. Chitov, Class. Quantum Grav. 15, 735 (1998); E. Elizalde and Yu.I. Shil'nov, hep-th/9809203; T. Inagaki, S.D. Odintsov, and Yu.I. Shil'nov, Int. J. Mod. Phys. A 14, 481 (1999).

[22] L. Dolan and R. Jackiw, Phys. Rev. D 9, 3320 (1974).

[23] D. Ebert and V.N. Pervushin, Teor. Mat. Fiz. (USSR) 36, 313 (1978); D. Ebert, L. Kaschluhn, and G. Kastelewicz, Phys. Lett. B 264, 420 (1991).

[24] M. Iwasaki and T. Iwado, Phys. Lett. B 350, 163 (1995).

[25] J. Berges and K. Rajagopal, Nucl. Phys. B538, 215 (1999); T.M. Schwarz, S.P. Klevansky, and G. Papp, Phys. Rev. C 60, 055205 (1999).

[26] K.G. Klimenko, Theor. Math. Phys. 89, 1161 (1992); K.G. Klimenko, Z. Phys. C 54, 323 (1992); K.G. Klimenko, A.S. Vshivtsev, and B.V. Magnitsky, Nuovo Cimento A 107, 439 (1994).

[27] V.P. Gusynin, V.A. Miransky, and I.A. Shovkovy, Phys. Rev. Lett. 73, 3499 (1994).

[28] K.G. Klimenko, IHEP 98-56, Protvino, 1998; hep-ph/9809218. 
[29] J. Schwinger, Phys. Rev. 82, 664 (1951).

[30] M.R. Brown and M.J. Duff, Phys. Rev. D 11, 2124 (1975); W. Dittrich, Fortschr. Phys. 26, 289 (1978).

[31] A.P. Prudnikov, Yu.A. Brychkov, and O.I. Marichev, Integrals and Series (Gordon and Breach, New York, 1986).

[32] A.S. Vshivtsev, K.G. Klimenko, and B.V. Magnitsky, Theor.
Math. Phys. 106, 319 (1996).

[33] H. Bateman and A. Erdeyi, Higher Transcendental Functions (McGraw Hill, New York, 1953).

[34] A.D. Balaev et al., Zh. Éksp. Teor. Fiz. 113, 1877 (1998) [JETP 86, 1026 (1998)]. 